\title{
Modulation of Barrier Properties of Monolayer Films from Blends of Polyethylene with Ethylene-co-Norbornene
}

\author{
S. Taglialatela Scafati, ${ }^{1}$ L. Boragno, ${ }^{2}$ S. Losio, ${ }^{1}$ S. Limbo, ${ }^{3}$ M. Castellano, ${ }^{4}$ \\ M. C. Sacchi, ${ }^{1}$ P. Stagnaro ${ }^{2}$ \\ ${ }^{1}$ ISMAC Milano, CNR, Istituto per lo Studio delle Macromolecole, Milano, Italy \\ ${ }^{2}$ ISMAC Genova, CNR, Istituto per lo Studio delle Macromolecole, Genova, Italy \\ ${ }^{3}$ Dipartimento di Scienze e Tecnologie Alimentari e Microbiologiche DISTAM, Università di Milano, Milano, Italy \\ ${ }^{4}$ Università di Genova, Dipartimento di Chimica e Chimica Industriale DCCI, Genova, Italy
}

Received 13 September 2010; accepted 9 December 2010

DOI 10.1002/app.33901

Published online 30 March 2011 in Wiley Online Library (wileyonlinelibrary.com).

\begin{abstract}
Flexible monolayer films with modulated diffusional and optical properties were achieved from blends of a LDPE/LLDPE matrix and an ethylene/norbornene copolymer (COC). A significant decrease of $\mathrm{O}_{2}$ and $\mathrm{CO}_{2}$ permeability was observed for cast films with $\mathrm{COC}$ content from 5 to $20 \mathrm{wt} \%$. SEM analysis showed that, despite the incompatibility of the blend components, good dispersion and distribution of the COC domains in the PE matrix were achieved; as a consequence, for COC content ranging from 5 to $10 \mathrm{wt} \%$ the film transparency results practically unchanged. The UV light transmission decreased on increasing the percentage of COC, which constitutes also a protection against UV radiations. A noticeable improve-
\end{abstract}

ment was observed in the mechanical properties: adding only $5 \mathrm{wt} \%$ of COC, the elastic modulus becomes five times higher than that of the PE matrix. The rheological behavior of the blends keeps practically unchanged with respect to the matrix, allowing to use the typical LDPE processing conditions. Sustainable inexpensive flexible films answering the packaging requirements of specific categories of food were achieved. (c) 2011 Wiley Periodicals, Inc. J Appl Polym Sci 121: 3020-3027, 2011

Key words: ethylene/norbornene copolymer; gas permeation; mechanical properties; barrier modulation; polyethylene blend; thin films

\section{INTRODUCTION}

Thank to their peculiar sealing properties, low cost and low reactivity, several types of polyethylene (PE) are of common use as food packaging materials, including low-density (LDPE), high-density (HDPE), linear low-density (LLDPE), and very low density (VLDPE) grades. The disadvantage with polyethylene and polyolefins in general is in that they provide relatively low barrier to gases, in particular to oxygen, as well as to flavors and aromas, ${ }^{1}$ and, consequently, they are not adequate to long shelf life requirements of specific categories of food.

Numerous studies aimed at improving the barrier properties of the polymers ${ }^{2-5}$ and a number of different technologies providing high barrier plastic films have been developed. ${ }^{6,7}$ A common route consists in combining materials capable of giving higher barrier than the individual components through lamination or coating. ${ }^{8}$ For flexible materi-

Correspondence to: P. Stagnaro (stagnaro@ge.ismac.cnr.it).

Contract grant sponsor: Regione Lombardia; contract grant number: 4161-MOD-IM-PACK.

Journal of Applied Polymer Science, Vol. 121, 3020-3027 (2011) (c) 2011 Wiley Periodicals, Inc. als, the traditional barrier layer consists in aluminum, in the form of a thin vacuum-deposited coating (metallization), ${ }^{9}$ which improves the film barrier up to a 40 -fold. ${ }^{10}$ Silica coatings have also received considerable attention: a thin layer of $\mathrm{SiO}_{2}$ applied on the film surface does provide significant enhancement in barrier properties. ${ }^{8}$

A second process commonly used to improve barrier properties of plastic films is co-extrusion. ${ }^{10} \mathrm{In}$ this way it is feasible to couple barrier properties typical of different materials. As an example, polyester films provide a better barrier to gases, such as oxygen and carbon dioxide, whereas polypropylene, polyethylene, and poly(vinylidene chloride) films are more effective against moisture. Many polymers, such as poly(vinyl alcohol) (PVOH), ethylene vinyl alcohol copolymer (EVOH), and most polyamides (Pas) exhibit good oxygen barrier only in the dry state. Thus, they have to be sandwiched between water vapor barrier films to maintain their function. ${ }^{9}$

Although such packaging solutions provide high barrier properties and are widely used, they have the disadvantage of a certain degree of complexity that makes these products not easy to recycle and/ or destroy at their life-end. Moreover, a total barrier is no longer a general goal of food packaging, but rather saving freshness is often required. This is the 
case, for example, of cheese and meat, which must be consumed within a limited number of days, in contrast to bakery products (e.g., cereals and biscuits), which are suppose to last several months. In other words, nowadays, the guide idea is that it is necessary to go over the simple concept of high barrier package to finalize the research efforts rather to modulate film permeability, in function of the specific requirements of the different foods.

In this work modulation of permeability is attained by melt blending a commercial polymer for packaging with a high-barrier material. Indeed, melt blending ${ }^{11,12}$ is a less complicated and therefore a less costly technology than, for example, lamination or coating. Moreover, monolayer films based on blends are attractive alternatives to multilayer co-extruded films. ${ }^{13}$

A typical LDPE/LLDPE blend was chosen as principal component. Such a "conventional" matrix was melt blended with an ethylene/norbornene copolymer (COC). COCs are high added value materials characterized by excellent transparency, high rigidity, and other interesting properties, such as good thermal stability and resistance to acids and bases, ${ }^{14}$ owing to their molecular and microstructural features. ${ }^{15}$ For instance, being these materials fully amorphous, high transparency and clarity of their manufacts is achieved; moreover, the high density of the amorphous phase provide them with high impermeablility to gases and vapors. In particular, the extremely high water vapor barrier and low moisture absorption ${ }^{16-18}$ make such materials interesting for this research.

Taking into account their high cost, the perspective of developing a competitive packaging monolayer film, by adding a limited percentage of COC in a low-cost polyethylene matrix, appears particularly attractive. In this way, it is possible to take advantage of the better features of both the blend components: high barrier of COC together with low-cost and processability of LDPE.

\section{EXPERIMENTAL}

\section{Materials}

The polyethylene (PE) matrix (60 wt \% $m$-LLDPE, 40 wt \% LDPE) was prepared with an ethylene/1octene copolymer from metallocene ( $m$-LLDPE, EXXON Mobil) and polyethylene from high pressure process (LDPE, Atofina). Erucamide $(0.3 \mathrm{wt} \%)$ was added as a processing aid. Ethylene/norbornene copolymer (COC) was kindly supplied by Ticona (norbornene $33 \mathrm{~mol} \%$, density $1.020 \mathrm{~g} / \mathrm{cm}^{3}, \mathrm{~T}_{\mathrm{g}} 80^{\circ} \mathrm{C}$ ).

\section{Preparation of blends}

The PE matrix/COC blends with different compositions $(95 / 5,90 / 10,85 / 15$, and $80 / 20 \mathrm{wt} / \mathrm{wt})$ were processed in a single-screw extruder (GiMac - Italy) with $\mathrm{L} / \mathrm{D}=22$, equipped with a Ross ISG static mixer, containing a sequence of 12 mixing elements. Operating conditions were: screw speed 50 and 80 rpm, cylinder temperature profile: $225,230,230,230$, $235^{\circ} \mathrm{C}$ and die exit temperature $225^{\circ} \mathrm{C}$. The PE matrix (60 wt \% m-LLDPE, $40 \mathrm{wt} \%$ LDPE) was prepared by using the same mixing conditions.

\section{Thermal analysis}

The thermal properties of PE matrix, COC, and their blends were analyzed using a Mettler-Toledo DSC $821^{\mathrm{e}}$ instrument. The thermograms were obtained with scanning rates of $20^{\circ} \mathrm{C} / \mathrm{min}$ and measurements were carried out in nitrogen atmosphere at a flow rate of $40 \mathrm{~mL} / \mathrm{min}$. All samples were treated as follows: after destroying the nascent crystallinity at $180^{\circ} \mathrm{C}$, the specimens were cooled under controlled conditions down to $-100^{\circ} \mathrm{C}$. A second heating run was imposed to acquire information on the melting behavior.

\section{Molecular characterization}

Molecular mass and molecular mass distribution of the starting materials were determined by a high temperature gel permeation chromatograph (GPCHT Waters 2000) that uses two on-line detectors: a differential viscometer (DV) and a differential refractometer (DRI) as concentration detector. The samples were dissolved in $o$-dichlorobenzene supplied by Aldrich-Chemie and the measurements were carried out at $145^{\circ} \mathrm{C}$ with a flow rate of $0.8 \mathrm{~mL} / \mathrm{min}$. The columns (3 TSK gel HT) were calibrated with narrow standards of polystyrene with molecular mass ranging from $162 \mathrm{~g} / \mathrm{mol}$ to $5.6 \times 10^{6} \mathrm{~g} / \mathrm{mol}$. The calibration curve for polystyrene was converted to polyethylene by using the Mark-Houwink constants for polyethylene.

\section{Rheological measurements}

The rheological parameters were determined on an AR2000 rotational rheometer produced by TA Instruments. The rheological measurements were carried out on $25 \mathrm{~mm}$ diameter disks, $1 \mathrm{~mm}$ thick, obtained by using a laboratory press. Parallel plate geometry ( $25 \mathrm{~mm}$ diameter and $500 \mu$ gap) was used for rheological experiments at $160^{\circ} \mathrm{C}$. Measurements in oscillation mode (frequency sweep test) were performed at $10 \%$ strain level with frequencies from 630 to $0.05 \mathrm{rad} / \mathrm{s}$ in nitrogen atmosphere.

\section{Cast film process}

Cast films were prepared by using a single screw extruder (Brabender 19/25/D) equipped with a UNIX 
TABLE I

Main Features of Starting Materials

\begin{tabular}{|c|c|c|c|c|c|}
\hline Material & $\begin{array}{l}\text { Comonomer type } \\
(\% \mathrm{~mol})^{\mathrm{a}}\end{array}$ & $\begin{array}{c}\mathrm{MFI}^{\mathrm{b}} \\
(\mathrm{g} / 10 \mathrm{~min})\end{array}$ & $\begin{array}{l}\text { Density }^{\mathrm{c}} \\
\left(\mathrm{g} / \mathrm{cm}^{3}\right)\end{array}$ & $\begin{array}{c}\bar{M}_{w}{ }^{\mathrm{d}} \\
(\mathrm{kg} / \mathrm{mol})\end{array}$ & $\bar{M}_{w} / \bar{M}_{n}{ }^{\mathrm{d}}$ \\
\hline$m$-LLDPE & 1-octene (4.9) & 3 & 0.902 & 68 & 2.1 \\
\hline LDPE & - & 0.8 & 0.924 & 104 & 5.5 \\
\hline $\mathrm{COC}$ & norbornene (33) & 6 & 1.020 & 83 & 2.4 \\
\hline
\end{tabular}

${ }^{\text {a }}$ Calculated by NMR.

${ }^{b}$ Melt Flow Index: $190^{\circ} \mathrm{C}, 2.16 \mathrm{~kg}$.

${ }^{\mathrm{c}}$ From data sheet.

d Measured by GPC.

take-off unit. 70-80 im thick, $10 \mathrm{~cm}$ wide films of PE matrix and PE matrix/COC blends were extruded with the following temperature profile: $T_{1}, T_{2}, T_{3}=$ 200, $T_{\text {head }}=210^{\circ} \mathrm{C}$. The temperature of the take-off rollers was set at $30^{\circ} \mathrm{C}$ and the screw rotation rate was $40 \mathrm{rpm}$.

\section{Morphological analysis}

The morphology of the blends and films were determined by scanning electron microscopy (SEM) using a Leica Stereoscan 440 at $20 \mathrm{kV}$ accelerating voltage. The observations were carried out on the fracture surfaces of the specimens, perpendicular to the traction direction. All samples were fractured in liquid nitrogen. The surfaces were gold-coated by a sputtering coating unit model AGAR PS3.

\section{Optical properties}

The haze and the UV-visible transmission spectra of the films were determined by using a Lambda 650 Perkin Elmer spectrophotometer equipped with a $150 \mathrm{~mm}$ integrating sphere. The haze was measured according to ASTM D1003. The transparency was determined according to ASTM D1746 by measurements at $550 \mathrm{~nm}$ with the same instrument without the integrating sphere.

\section{Permeability tests}

Diffusion properties of films, described as oxygen permeability $\left(\mathrm{KPO}_{2}\right)$ and carbon dioxide permeability $\left(\mathrm{KPCO}_{2}\right)$ were determined by using, in the first case, a Lyssy isostatic permeability tester OPT 5000, and, in the latter case a Lyssy quasi-isostatic permeability tester GPM 500 connected to a GL Science gas chromatograph GC320.

The first apparatus is a permeability cell consisting of two cylindrical stainless steel compartments separated by a sample film $(10 \times 10 \mathrm{~cm})$. The permeant and inert carrier $\left(\mathrm{N}_{2}\right)$ gases flow continuously through the compartments. This method is called isostatic since the same total pressure is achieved on both sides of the film specimen by balancing the two gas flows in order to avoid film deformation. A partial pressure difference $\Delta p$ is also maintained, thereby providing the constant driving force for the permeant gas to move across the film.

In the quasi-isostatic method the lower compartment is sealed and filled with He up to a certain pressure. In the upper compartment, a stream of permeant gas creates the driving force for the permeant molecules to move through the film and accumulate in the lower chamber. The permeation rate reaches the steady state when the equilibrium between the two partial pressures of the permeant is achieved. Since the film area $A$ and pressure difference $\Delta p$ are known, the permeance can be calculated. ${ }^{19}$ The permeability values were determined at $23^{\circ} \mathrm{C}, 0 \% \mathrm{RH}$ (Relative Humidity), as indicated by the ASTM D1434 - 82 (2009). ${ }^{20}$

\section{Mechanical tests}

The tensile properties of the cast films were determined with a Zwick/Roell Z010 model according to ASTM D-882 for thin plastic sheets. Elastic modulus and elongation at break were measured at $23^{\circ} \mathrm{C}$. Analyses were carried out with initial speed of 10 $\mathrm{mm} / \mathrm{min}$ and crosshead speed of $500 \mathrm{~mm} / \mathrm{min}$.

\section{RESULTS AND DISCUSSION}

\section{Blend preparation and characterization}

Blends of a PE matrix and ethylene/norbornene copolymer (COC) at different COC contents were prepared by melt blending to improve the barrier properties of plastic films for packaging. As PE matrix, a blend of $m$-LLDPE and LDPE $(60 / 40 \mathrm{wt} / \mathrm{wt})$ was chosen, to combine the good mechanical properties of the $m$-LLDPE with the easy processability of LDPE. ${ }^{21}$ Table I reports the composition and molecular characterization of the starting materials.

Figure 1 depicts the complex viscosity of neat LDPE and $m$-LLDPE, and of their blend (PE matrix) as a function of the angular frequency at $160^{\circ} \mathrm{C}$. In the case of neat LDPE, a shear thinning behavior is 


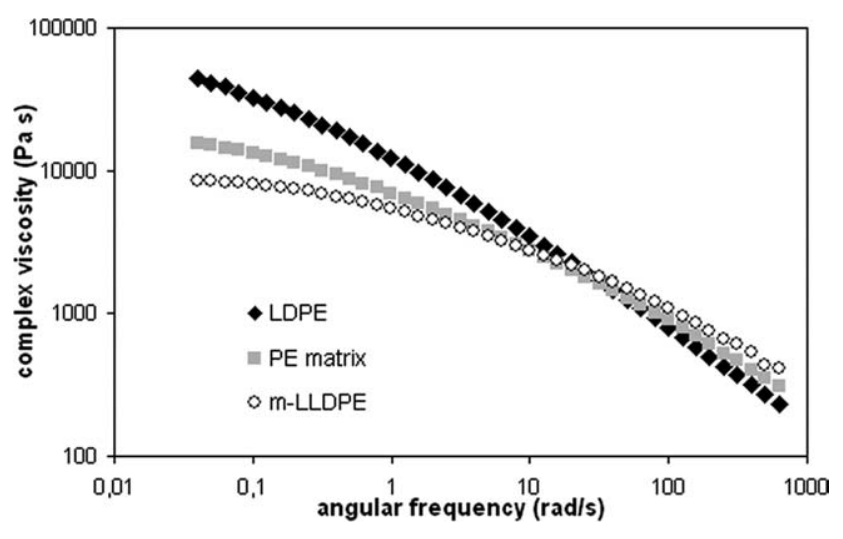

Figure 1 Complex viscosity versus angular frequency at $160^{\circ} \mathrm{C}$ of neat LDPE, neat $m$-LLDPE and PE matrix.

observed as the combined effect of polydispersity and high degree of long chain branching. ${ }^{22}$ As expected, PE matrix shows a value of complex viscosity intermediate between those of the two components: this is indicative of their good miscibility. ${ }^{23}$

Table II shows the results of the DSC measurements carried out on the PE matrix/COC blends at different COC contents.

This study has shown that the presence of amorphous COC $\left(T_{\mathrm{g}}=80^{\circ} \mathrm{C}\right)$ has no effect on the thermal properties of the PE matrix. In fact, the melting temperatures $T_{\mathrm{m}}$ and crystallization temperatures $T_{\mathrm{c}}$ of the blends are independent of the COC content. This behavior suggests a complete immiscibility of the two components in the molten state, due to the high content $(33 \mathrm{~mol} \%)$ of norbornene co-units in COC that do not allow significant interactions with the PE matrix.

The rheological behavior of the blends was studied and the complex viscosity curves are shown in Figure 2 as a function of frequencies; the curves relative to PE matrix and COC are reported for comparison. The different blends show very similar behavior: taking the PE matrix as a reference point, a slight increase of complex viscosity with increasing of COC content is observed.

These results confirm the immiscibility of the blend components and show that the presence of COC has no influence on the PE matrix rheological properties. As a consequence, the processing param-

TABLE II

Thermal Properties of PE Matrix and Blends

\begin{tabular}{lcccc}
\hline \multicolumn{1}{c}{ Sample } & $T_{\mathrm{c}}{ }^{\circ} \mathrm{C}$ & $\Delta H_{\mathrm{c}}(\mathrm{J} / \mathrm{g})$ & $T_{\mathrm{m}}{ }^{\mathrm{a}}\left({ }^{\circ} \mathrm{C}\right)$ & $\Delta H_{\mathrm{m}}{ }^{\mathrm{a}}(\mathrm{J} / \mathrm{g})$ \\
\hline PE matrix & 90.8 & 100 & 108.8 & 97 \\
PE matrix/COC 95/5 & 90.0 & 106 & 108.4 & 111 \\
PE matrix/COC 90/10 & 90.3 & 106 & 108.9 & 109 \\
PE matrix/COC 85/15 & 90.9 & 99 & 108.4 & 108 \\
PE matrix/COC 80/20 & 91.0 & 99 & 107.6 & 108 \\
\hline
\end{tabular}

${ }^{\text {a }}$ Measured on second heating.

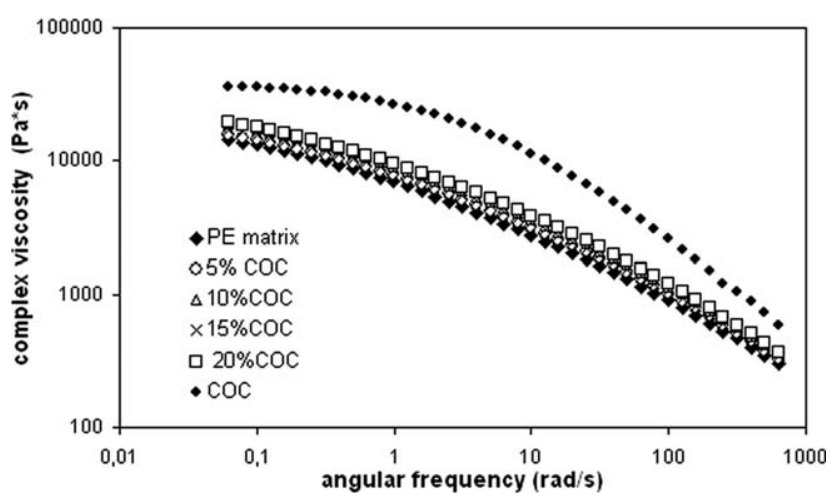

Figure 2 Rheological behavior of blends PE matrix/COC and pure materials (PE matrix, COC).

eters adopted for the PE matrix are expected to be adequate for the PE matrix/COC blend as well.

\section{Blend and film morphology}

A morphological study was carried out on the blends as well as on the corresponding films obtained by casting. The SEM micrographs of the pellets of the PE matrix/COC $80 / 20$ blend and of the corresponding film, are reported in Figure $3(a, b)$,
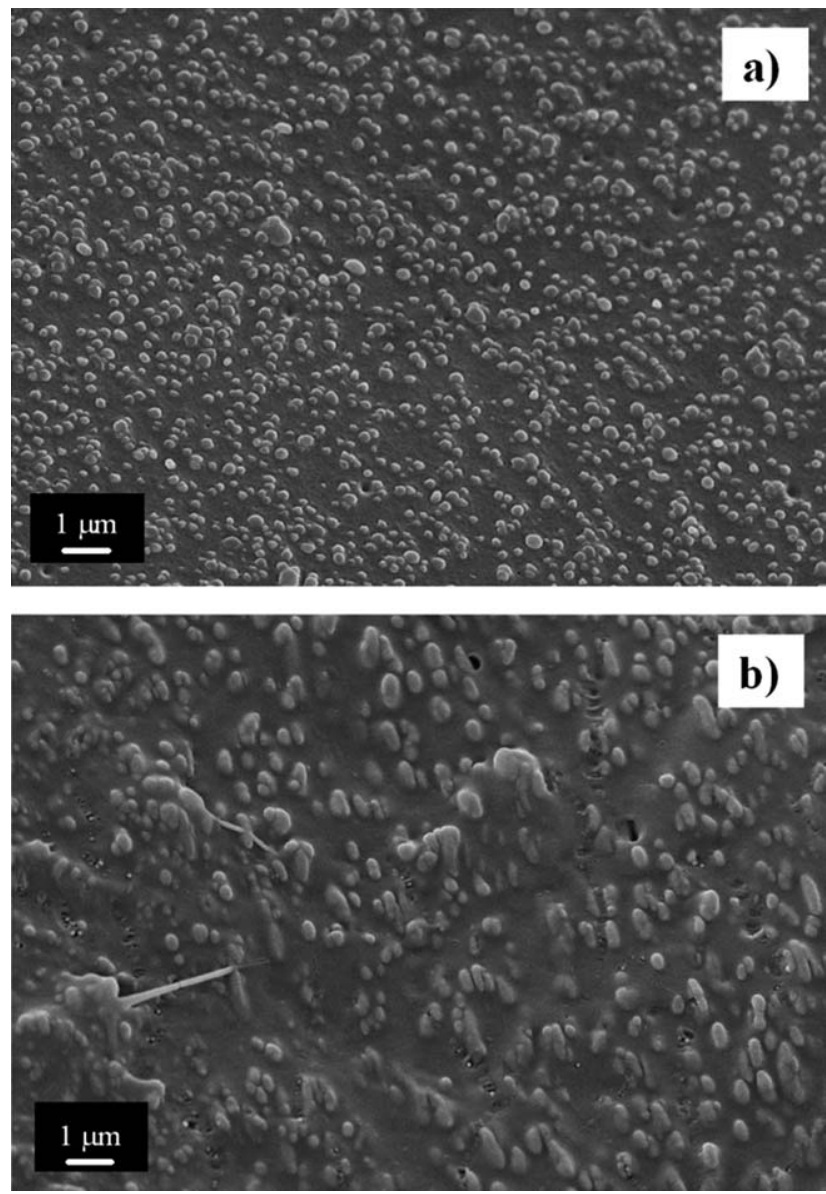

Figure 3 SEM micrographs of PE matrix/COC 80/20 pellet (a) and film (b). 

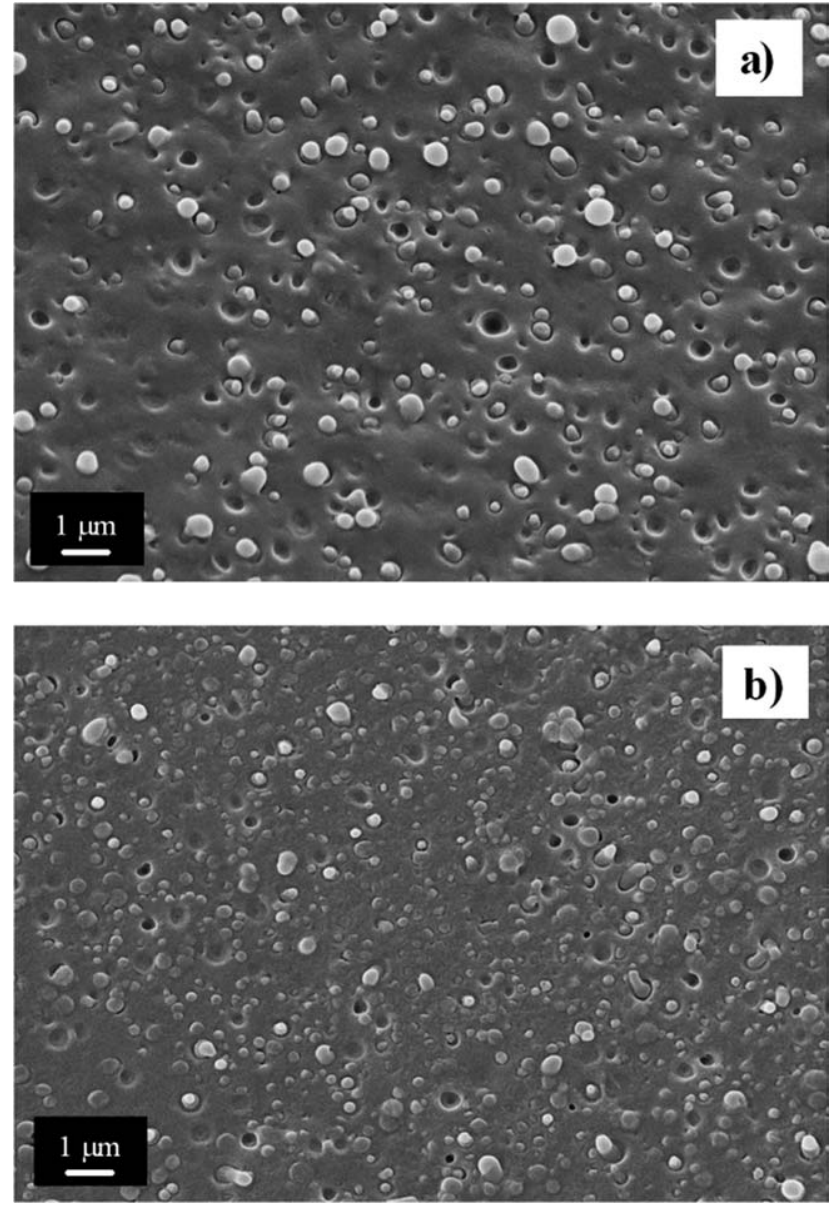

Figure 4 SEM micrographs at different COC content: $10 \%$ (a) and $20 \%(b)$.

respectively. Such an analysis provides visual evidence of the above discussed immiscibility of the components: indeed, COC appears dispersed into the PE matrix in the form of slightly elongated heterophasic domains with diameter ranging from 100 to $300 \mathrm{~nm}$.

The morphology of the film is similar to that of the corresponding pellets; however, one can observe a certain coalescence of the COC domains in the film, as clearly evident from the slight increase in their size. The COC dispersion in the PE matrix appears not to be influenced by the composition: the higher content of COC leads to a greater number of domains of substantially comparable size. As an example, SEM micrographs of samples with 10 and 20 wt \% COC content are shown in Figure $4(a, b)$, respectively.

The differences on morphology due to different screw rotational speeds (50 and $80 \mathrm{rpm}$ ) were evaluated for blend PE matrix/COC 90/10 (Fig. 5).

One can see that a higher speed of the screw (and consequently a higher shear stress applied to the material) leads to the formation of slightly deformed domains and of a slight coalescence of the particles of COC dispersed in the matrix.

SEM micrographs show that, despite the incompatibility of the blend components, good dispersion and distribution are achieved for all the blends, applying suitable processing conditions. Furthermore, morphological analyses allowed us to explain the increasing haze experienced on increasing COC content: in facts, the size of the larger particles in the more concentrated blends are of the same magnitude of the visible radiation wavelength, thus affecting the light transmission within the film bulk. This also affects the film clarity, accounting for the slight decrease of transparency of the films containing larger particles.

\section{Film optical properties}

The optical properties of plastic films for food packaging applications are of great importance not only as far as the quality preservation of the food is concerned, but also from an aesthetic point of view. From one hand, it should be desirable to have films with high barrier performances against UV
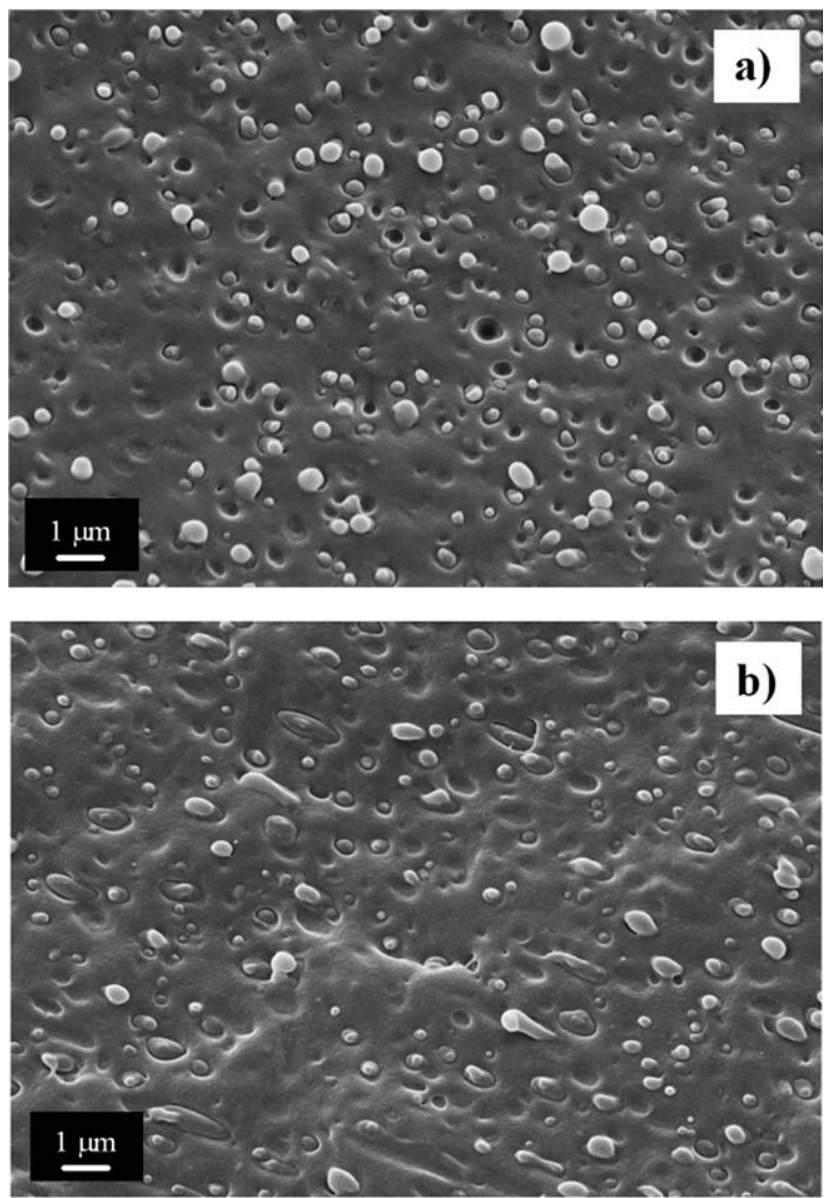

Figure 5 SEM micrographs of PE matrix/COC 90/10 prepared by single-screw at $50 \mathrm{rpm}$ (a) and $80 \mathrm{rpm}$ (b). 

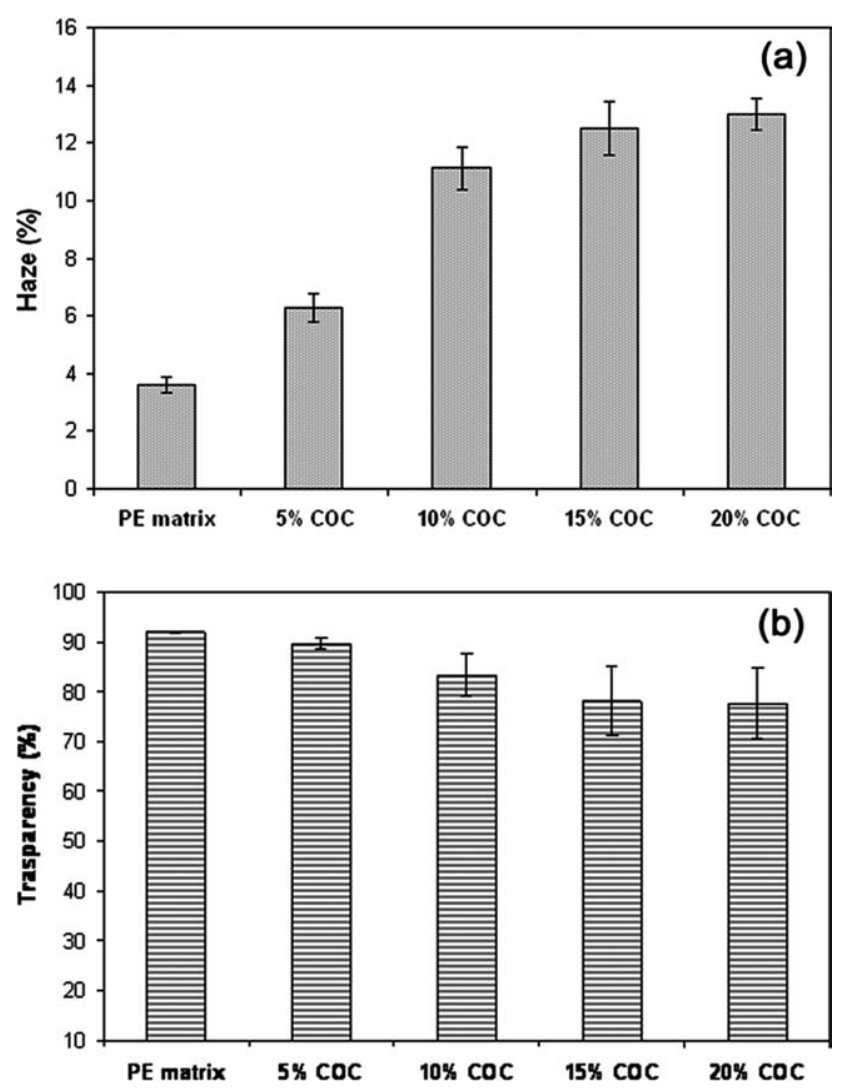

Figure 6 Optical properties: haze (a) and transparency (b) as a function of COC content.

radiations to avoid damaging reactions, however, industries are always looking toward transparent films, due to the increasing consumer demand to "see through" the package. Taking into account these considerations, this research also dealt with the influence of COC content on some optical characteristics of the films obtained from the blends.

\section{Haze and transparency}

For transparent polymer films direct transmittance is defined as the fraction of the incident light at a specified wavelength that emerges not deviated from the other side of the film. In the case of films from blends of completely miscible polymers light scattering does not occur and transparency is maintained. However, in blends where the minor component forms isolated domains dispersed into the matrix light scattering is observed, due to the difference in the refractive indices of the two phases. $^{24}$ This light scattering is responsible for haze, that is the cloudy or turbid appearance of an otherwise transparent specimen. Haze is defined as the percentage of light that is deflected more than $2.5^{\circ}$ from the incident light direction.

In this respect, the morphology above observed for the PE matrix/COC blends is likely to have an influence on the optical properties of the films. The haze of the PE matrix based films increases with COC content, as expected [Fig. 6(a)]. Conversely, the transparency, measured as direct transmittance at $550 \mathrm{~nm}$, decreases with increasing COC content [Fig. 6(b)]. However, it is worthwhile to stress that with COC content ranging from 5 to $10 \mathrm{wt} \%$ the film transparency results practically unchanged, and even with 20 wt \% COC content a decrease of transparency lower than $18 \%$ is observed.

\section{UV transmission properties}

Most degradation reactions of polymeric matrices as well as of packed food are due to exposure to ultraviolet radiation which, being characterized by a higher energy than visible radiation, induces chemical bond breaking and formation of highly reactive radical species. The role of UV light is not negligible because even small amount of this radiation can promote the formation of free radicals from lipids leading to photolytic autoxidation. On the basis of these considerations, it seemed appropriate to evaluate UV-visible transmission spectra of the prepared films. The influence of COC content on UV transmission is reported in Figure 7. One can observe that the UV radiation transmission noticeably decreases with increasing COC concentration. Thus, the presence of $\mathrm{COC}$ can constitute a protection of the packed food against UV light. Interestingly, the UVabsorbing behavior of the blend with $10 \mathrm{wt} \% \mathrm{COC}$ content is very close to that of the blends richer in COC. Consequently, it is possible to produce films with noticeable "UV-blocking" capability but still endowed with the desirable transparency.

\section{Barrier properties}

Oxygen and carbon dioxide barrier properties have been studied for the films deriving from the blends

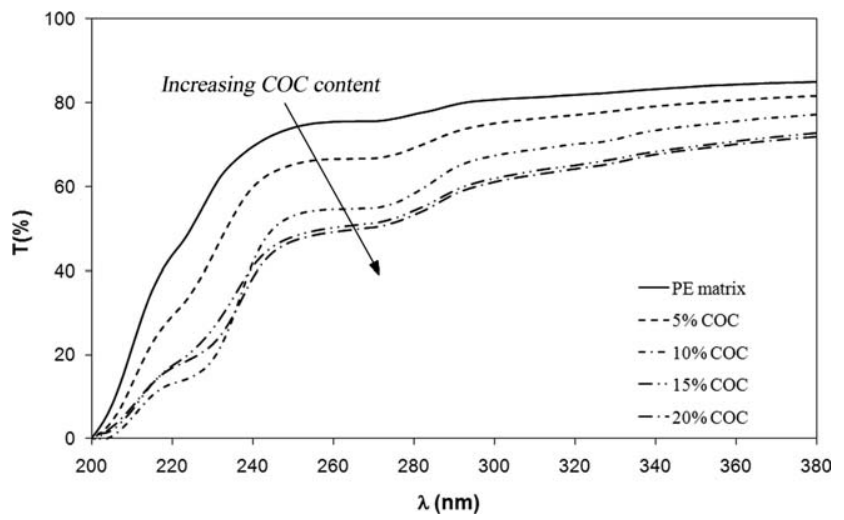

Figure 7 Transmission spectra of all the blends and the PE matrix. 

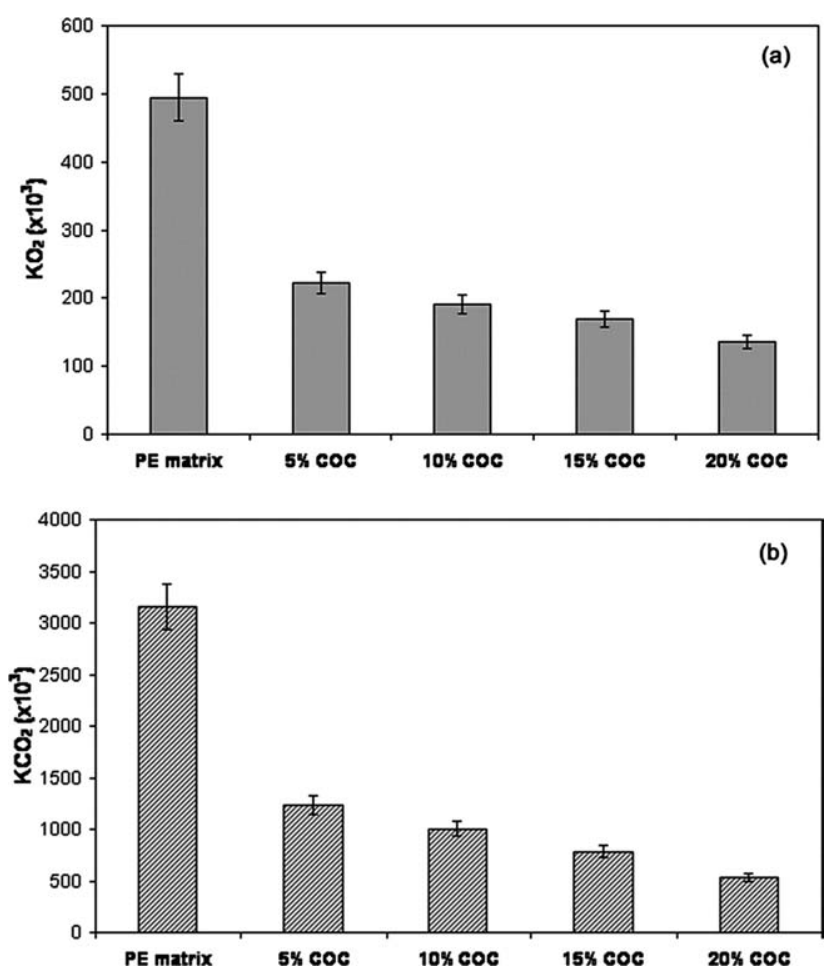

Figure $8 \quad \mathrm{O}_{2}$ (a) and $\mathrm{CO}_{2}$ (b) permeability coefficients of monolayers films as a function of COC content.

and compared with those of the matrix. The evolution of the $\mathrm{O}_{2}$ and $\mathrm{CO}_{2}$ permeability coefficients as a function of the COC content is shown in Figure $8(\mathrm{a}, \mathrm{b})$, respectively. As it can be seen, the desired effect on film barrier is achieved. Indeed, the addition of $\mathrm{COC}$ led to a drastic and progressive decrease of both $\mathrm{O}_{2}$ and $\mathrm{CO}_{2}$ permeability constants with respect to those of the PE matrix. In particular, the oxygen permeability coefficients falls to about $45 \%$ of the reference value (from 494,000 to 222,000 $\mathrm{cm}^{3} \cdot \mu / \mathrm{m}^{2} \cdot 24 \mathrm{~h} \cdot$ bar) due to a COC content as low as 5 wt $\%$, while, for 20 wt \% content, $\mathrm{KPO}_{2}$ is $27 \%$ of the corresponding value of the PE matrix. The carbon dioxide constant permeability reduces to about $39 \%$ of the PE matrix value (from 3,150,000 to $1,004,000 \mathrm{~cm}^{3} \cdot \mu / \mathrm{m}^{2} \cdot 24 \mathrm{~h} \cdot$ bar) with $5 \mathrm{wt} \%$ content of COC, while, for $20 \mathrm{wt} \%$ content, $\mathrm{KPCO}_{2}$ is $17 \%$. Therefore, the selectivity $\left(\mathrm{KPCO}_{2} / \mathrm{KPO}_{2}\right)$ is also sensibly affected by the addition of $\mathrm{COC}$ to the matrix.

Gas permeation is a three step process: atmospheric gases are first adsorbed onto the film surface, then diffuse within the bulk and are finally released from the opposite surface by desorption of the gas molecules. ${ }^{25}$ Melt blending of a high barrier polymer such as a COC should mainly affect the diffusion step, while negligible effects are expected on the adsorption and desorption processes. To account for the observed barrier increase phenomenon, we hypothesize that the well-dispersed, high dense
COC domains force the gas molecules to follow a longer (tortuous) path to avoid the nonpermeable COC islands, This phenomenon would be similar to the well-known effect that filler nanoparticles have on gas permeability in polymeric nanocomposites. ${ }^{26-28}$

Obviously, the obtained permeability values are not comparable with those of metallized layers that, although not providing a total barrier against gases and vapors, are anyhow able of reducing the permeation coefficient to values lower than $50-70 \mathrm{~cm}^{3} \cdot \mu / \mathrm{m}^{2} \cdot 24 \mathrm{~h} \cdot$ bar. $^{9}$

In spite of this, the films here studied can be exploited in food industry since the modulation of permeation is pursued for different applications which do not require a total barrier but only a certain reduction of gas transport, especially if the shelf life of food product is short. In addition, the proposed approach allow to maintain already established processing conditions while modestly affecting the product cost and facilitating end-use recycle.

The above results are of great interest due to the possibility of improving at the same time both the barrier properties and the UV-absorbing behavior of a monolayer polyolefinic film by varying the COC content within 5-10 wt \%, that is, in the range in which the transparency of the film is practically unaffected.

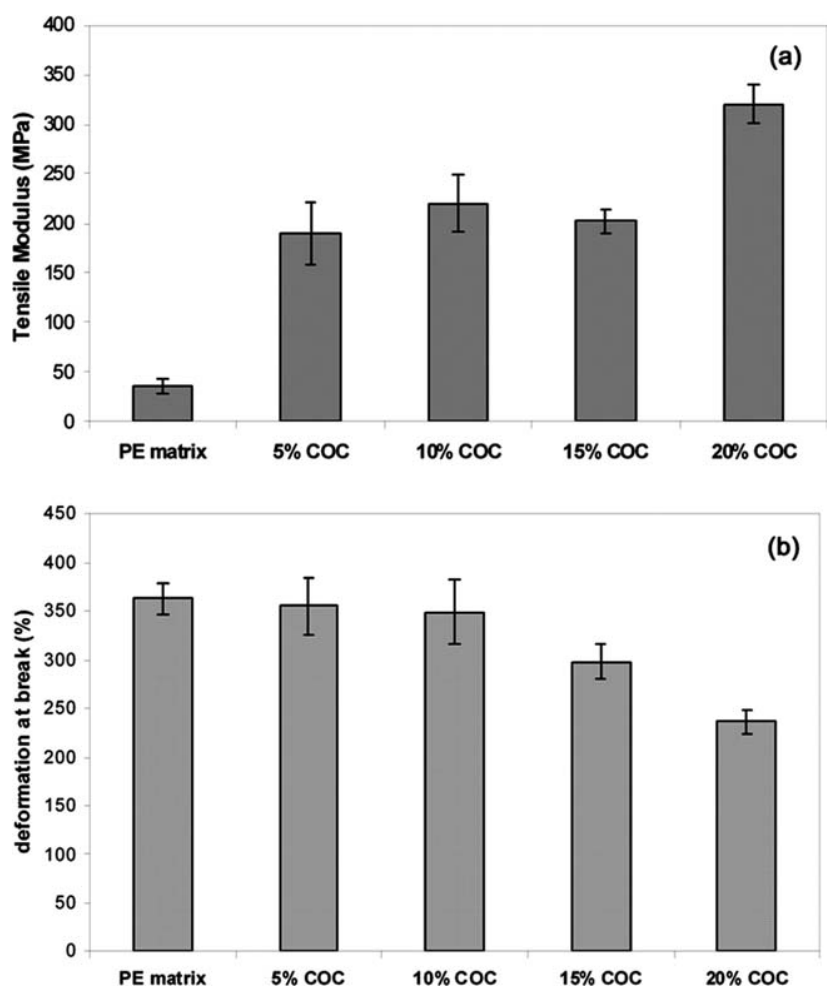

Figure 9 Elastic modulus (a) and deformation at break (b) of films vs. COC content. 


\section{Mechanical properties}

The study of the mechanical properties of the PE based films also revealed important improvements conferred by the presence of COC. Indeed, tensile tests showed that the presence of COC causes a remarkable increase of elastic modulus, and a decrease of deformation at break, as evidenced in Figure 9. In particular, the elastic modulus of the films increases with increasing concentration of COC: from about 40 $\mathrm{MPa}$ of the PE matrix film to $320 \mathrm{MPa}$ of the PE matrix/COC $80 / 20$ film. What is interesting is that, by adding only $5 \mathrm{wt} \%$ of $\mathrm{COC}$, the elastic modulus becomes five times higher than that of the PE matrix, while the deformation at break is almost unchanged. These results show that the presence of a small amount of COC can strongly improve the mechanical performances of the original polymer without affecting the structural properties.

\section{CONCLUSION}

Through melt blending of COC with a commonly used PE-based matrix, we attained the tuning of the diffusional properties of monolayer films for packaging applications. A significant decrease of $\mathrm{O}_{2}$ and $\mathrm{CO}_{2}$ permeability is observed with $\mathrm{COC}$ content ranging from 5 to $20 \mathrm{wt} \%$ : the desirable value of the diffusional properties can be obtained by adding the proper amount of COC. In the UV region, the light transmission progressively decreases on increasing the percentage of $\mathrm{COC}$, which as a consequence produces, in addition to the influence on gas permeability, a significant protection against UV radiations. What is interesting is that such results are obtained by varying the COC content in the range in which the films are still highly transparent.

Clearly, such an approach cannot intrinsically lead to a barrier as high as those obtained through commonly used procedures, such as lamination or coextrusion. These new packaging solutions are destined to specific sectors of food products that must be consumed within a relatively short time and whose shelf-life needs to be limitedly improved. It is also worthwhile to note that such results are obtained with monolayer films, produced with a quite simple technology, and that the barrier improvement is achieved by melt blending rather than with the usual time-consuming and scarcely sustainable processes.

\section{References}

1. Risch, S. J. J. Agr Food Chem 2009, 57, 8089.

2. Hu, Y. S.; Prattipati, V.; Mehta, S.; Schiraldi, D. A.; Hiltner, A.; Baer, E. Polymer 2005, 46, 2685.

3. Jarus, D.; Hiltner, A.; Baer, E. Polymer 2002, 43, 2401.

4. Andrio, A.; Cabot, C.; Atmandi, H. Polym Test 2004, 23, 475.

5. Mrkiæ, S.; Galiæ, K.; Ivankoviæ, M. J. Plastic Film Sheeting 2007, 23, 239.

6. Strupinsky, G.; Brody, A.A twenty-year retrospective on plastics: oxygen barrier packaging materials - TAPPI Polymers, Laminations and Coating Conf. Proceed.; San Francisco, CA, 1998; p 119.

7. Silvis, H. C. Trends Polym Sci 1997, 5, 75.

8. Risch, S. J. Abstr Pap Amer Chem Soc 1999, 218, U733.

9. Lange, J.; Wyser, Y. Package Technol Sci 2003, 16, 149

10. Hanlon, J. F. Handbook of Package Engineering, 2nd ed; Technomic: Lancaster, PA, 1992.

11. Lee, S. Y.; Kim, S. C. Polym Eng Sci 1997, 37, 463.

12. Brown, W. E. Plastics in Food Packaging: Properties, Design, and Fabrication; Dekker: New York, 1992; 313.

13. Trongsatitkul, T.; Duangdao, A.; Chinsirikul, W. Macromol Symp 2004, 216, 265.

14. Kolarýk, J.; Krulis, Z.; Slouf, M.; Fambri, L. Polym Eng Sci 2005, 45, 817.

15. Tritto, I.; Boggioni, L.; Jansen, J. C.; Thorshaug, K.; Sacchi, M. C.; Ferro, D. R. Macromolecules 2002, 35, 616.

16. Brochures available at Topas Advanced Polymer site: http:// www.topas.com/news-literature-brochures (downloaded 23rd November 2010)

17. Lamonte, R. R.; McNally, D. Adv Mat Proc 2001, 159, 33.

18. Forcinio, H. Pharm Tech June 2001, 22.

19. Lee, S. L.; Yam, K. L.; Piergiovanni, L. Food Packaging Science and Technology; CRC Press: New York, 2008; p 79.

20. ASTM D1434 - 82 (2009). Standard Test Method for Determining Gas Permeability Characteristics of Plastic Film and Sheeting

21. Hussein, I. A.; Hameed, T. J. Appl Polym Sci 2005, 97, 2488.

22. Delgadillo-Velazquez, O.; Hatzikiriakos, S. G.; Sentmanat, M. J Polym Sci Part B: Polym Phys 2008, 46, 1669.

23. Fang, Y.; Carreau, P. J.; Lafleu, P. G. Polym Eng Sci 2005, 45, 1254.

24. Maruhashi, Y.; Iida, S. Polym Eng Sci 2001, 41, 1987.

25. Piergiovanni, L.; Limbo, S. Food Packaging; Springer: Italia, Chapter IV.

26. Ou, C.-F.; Hsu, M.-C. J Polym Res 2007, 14, 373.

27. Bharadwaj, R. K. Macromolecules 2001, 34, 9189.

28. Sinha Ray, S.; Okamoto, M. Prog Polym Sci 2003, 28, 1539. 